\title{
Abdominal muscle activity during breathing in different postural sets in healthy subjects
}

\author{
Antonio Mesquita Montes, MSc, PT ${ }^{\mathrm{a}, \mathrm{b}}$, Sara Gouveia, PT ${ }^{\mathrm{c}}$, \\ Carlos Crasto, MSc, PT ${ }^{\mathrm{a}}$, Cristina Argel de Melo, PhD, PT a , \\ Paulo Carvalho, PhD, PT ${ }^{\text {a }}$, Rita Santos, MSc, PT ${ }^{\mathrm{a}}$, \\ João Paulo Vilas-Boas, PhD ${ }^{\text {b }}$
}

\footnotetext{
a Department of Physiotherapy, and Activity and Human Movement Study Center (CEMAH), School of Allied Health Technologies, Polytechnic Institute of Porto, Rua Valente Perfeito 322, 4400-330, Vila Nova de Gaia, Portugal

b Faculty of Sport, CIFI2D, and Porto Biomechanics Laboratory (LABIOMEP), University of Porto, Rua Dr. Plácido Costa 91, 4200-450, Porto, Portugal

c Private Practice, Portugal
}

\section{KEYWORDS}

Respiration;

Postural control; Core abdominal;

Body position;

Surface

electromyographic

activity

\begin{abstract}
Summary Objective: This study aims to evaluate the effect of different postural sets on abdominal muscle activity during breathing in healthy subjects.

Methods: Twenty-nine higher education students ( $20.86 \pm 1.48$ years; 9 males) breathed at the same rhythm (inspiration: $2 \mathrm{~s}$; expiration: $4 \mathrm{~s}$ ) in supine, standing, tripod and 4-point-kneeling positions. Surface electromyography was performed to assess the activation intensity of rectus abdominis, external oblique and transversus abdominis/internal oblique muscles during inspiration and expiration.

Results: During both breathing phases, the activation intensity of external oblique and transversus abdominis/internal oblique was significantly higher in standing when compared to supine $(p \leq 0.001)$. No significant differences were found between tripod position and 4-point-kneeling positions. Transversus abdominis/internal oblique activation intensity in these positions was higher than in supine and lower than in standing.

Conclusions: Postural load and gravitational stretch are factors that should be considered in relation to the specific recruitment of abdominal muscles for breathing mechanics.
\end{abstract}




\section{Introduction}

Postural control is the ability to maintain the stability of the body and its segments in response to internal and external forces that threaten to disturb the body's equilibrium (Horak et al., 1997). The central nervous system (CNS) interprets and organizes sensory inputs from the vestibular, visual and proprioceptive systems and the information received from the receptors that are located in and around joints to provide postural control. As a result of this weighting, the CNS detects and predicts instability and responds with the appropriate output (Balasubramaniam and Wing, 2002; Lackner and DiZio, 2005), wherein the abdominal muscles preserve the postural equilibrium and spinal stability by modulating the intra-abdominal pressure (Cholewicki et al., 1999). This modulation occurs as a result of the coordination of the activity of the abdominal, pelvic floor and diaphragm muscles (Hodges et al., 1997; Hodges and Gandevia, 2000a).

Nevertheless, the CNS modulates the motor activities of these trunk muscles during both postural and respiratory functions (Hodges, 1999) to concurrently regulate the intraabdominal and intra-thoracic pressures (Hodges et al., 2001). Although the activity of rectus abdominis (RA) and external oblique (EO) muscles is not respiration-related modulation, the tonic activity of diaphragm and transversus abdominis (TrA) muscles for postural control is modulated with the respiratory phasic activity (Hodges and Gandevia, 2000a, 2000b).

Despite their predominantly expiratory action, abdominal muscles contribute significantly to inspiration through their tonic activity, promoting a direct facilitation of diaphragm muscle contraction by preventing its excessive shortening during inspiration (Goldman et al., 1987). TrA muscle, due to its circumferential arrangement, has the most appropriate mechanical efficiency to perform this role (De Troyer et al., 1990). Moreover, the increased intra-abdominal pressure generated through the abdominal muscle contraction during expiration prepares the respiratory system for the next inspiration by optimizing the length-tension relationship of diaphragm muscle fibres (De Troyer and Estenne, 1988).

Regarding the abdominal muscles' dual task, the change of body orientation in space alters the configuration and the length of abdominal muscles and, consequently, the ability of respiratory muscles to act during breathing (De Troyer, 1983). Such modifications in mechanical efficiency may be due to the action of gravity on the abdominal content and wall, which affects the abdominal compliance and induces a change in the length of diaphragm muscle fibres (Estenne et al., 1985) and, consequently, in functional residual capacity (Dean, 1985). Nevertheless, the impact of different postural sets on abdominal muscle activity for the synchronization of postural and respiratory functions is not yet clear. Although the abdominal muscle activity increases from supine to standing (Abe et al., 1996; Barrett et al., 1994; De Troyer, 1983), there is little evidence regarding the individual recruitment of abdominal muscles in postural sets often assumed for unloading of the respiratory system, such as in tripod position (Booth et al., 2014). Furthermore, the four-point kneeling position, which facilitates the co-contraction of deep abdominal muscles - TrA muscle and lower fibres of internal oblique (IO) muscle - and back muscles - deep fibres of lumbar multifidus - (Hides et al., 2004) may be performed to improve the breathing mechanics. Thus, the aim of the present study was to evaluate the effect of different postural sets on abdominal muscle activity during breathing in healthy subjects. Specifically, the activation intensity of RA, EO and transversus abdominis/internal oblique (TrA/IO) muscles, during inspiration and expiration, were analysed in supine, tripod, four-point kneeling and standing positions.

\section{Methods}

\section{Sample}

A repeated measures study design was conducted with a sample composed by twenty-nine healthy higher education students who volunteered to participate in this research (9 males). Demographic and anthropometric data regarding the sample are described in Table 1. Participants were aged between 18 and 24 years and had not participated in aerobic physical activities with a moderate intensity (a minimum of 30 min on five days a week) or/and in aerobic physical activities with a vigorous intensity (a minimum of 20 min on 3 days a week), for a period exceeding one year (Thompson, 2014). Exclusion criteria included body mass index higher than $25 \mathrm{~kg} \mathrm{~m}^{-2}$; chronic nonspecific lumbopelvic pain (recurrent episodes of lumbopelvic pain for a period longer than three months); scoliosis, length discrepancy of the lower limbs or other postural asymmetries; history of spinal, gynaecological or abdominal surgery in the previous year; neurological or inflammatory disorders; metabolic or cardio-respiratory diseases; pregnancy or post-delivery in the previous six months; smoking habits; long-term corticosteroid therapy; and any conditions that may interfere with the data collection (American Thoracic Society/European Respiratory, 2002; Beith et al., 2001; Chanthapetch et al., 2009; Hermens et al., 2000; Mew, 2009; Reeve and Dilley, 2009). Each participant provided written informed consent, according to the Declaration of Helsinki. The anonymity of participants and the confidentiality of data were guaranteed. The Institutional Research Ethics Committee previously approved this study.

\section{Instruments}

Surface electromyography (sEMG) was performed to bilaterally assess the muscle activity of TrA/IO, EO, RA and erector spinae $(\mathrm{ES})$. The muscle activity was collected using

Table 1 Sample characterization: demographic and anthropometric data, with mean, standard deviation, minimum and maximum.

\begin{tabular}{lllll}
\hline & Mean & $\begin{array}{l}\text { Standard } \\
\text { deviation }\end{array}$ & Minimum & Maximum \\
\hline Age (years) & 20.86 & 1.48 & 18 & 24 \\
Body mass $(\mathrm{kg})$ & 59.38 & 10.79 & 44 & 85 \\
Height $(\mathrm{m})$ & 1.66 & 0.11 & 1.52 & 1.89 \\
BMI $\left(\mathrm{kg} \mathrm{m}^{-2}\right)$ & 21.34 & 1.77 & 18.34 & 24.45 \\
\hline
\end{tabular}

BMI body mass index. 
the BioPlux research device (Plux wireless biosignals S.A., Arruda dos Vinhos, Portugal) with analogue channels of 12 bits and a sampling frequency of $1000 \mathrm{~Hz}$, using double differential electrode leads. Disposable, self-adhesive $\mathrm{Ag} / \mathrm{AgCl}$ dual snap electrodes (Noraxon Corporate, Scottsdale AZ, United States of America) were used for the sEMG. The electrode characteristics were $4 \times 2.2 \mathrm{~cm}$ of adhesive area, $1 \mathrm{~cm}$ diameter of each circular conductive area and $2 \mathrm{~cm}$ of inter-electrode distance. These electrodes were connected to bipolar active sensors emgPLUX with a gain of 1000 , an analogue filter at $25-500 \mathrm{~Hz}$ and a common-mode rejection ratio of $110 \mathrm{~dB}$. The reference electrode used was a disposable self-adhesive $\mathrm{Ag} / \mathrm{AgCl}$ snap electrode (Noraxon Corporate, Scottsdale AZ, United States of America) for the SEMG, with $3.8 \mathrm{~cm}$ diameter of circular adhesive area and $1 \mathrm{~cm}$ diameter of circular conductive area. The sensors were Bluetooth connected through the sEMG device to a laptop. MonitorPlux software, version 2.0, was used to display and acquire the sEMG signal. An electrode impedance checker was used to assess the impedance level of skin (Noraxon Corporate, Scottsdale AZ, United States of America).

A respiratory flow transducer TSD117 - Medium Flow Trans $300 \mathrm{~L} \mathrm{~min}^{-1}$ connected to an amplifier DA100C General Purpose Transducer Amplifier Module, was used to detect both breathing phases. The respiratory flow was collected using the Biopac MP100WSW Data Acquisition System device (Biopac Systems Inc., Goleta CA, United States of America) with a sampling frequency of $100 \mathrm{~Hz}$. A bacterial filter AFT1 - Disposable Bacterial Filter, $22 \mathrm{~mm}$, a mouthpiece AFT2 - Disposable Mouthpiece, $22 \mathrm{~mm}$ and a nose clip AFT3 - Disposable Noseclip were also used. Acqknowledge software, version 4.1, (Biopac Systems Inc., Goleta CA, United States of America) was used to display and acquire the respiratory flow signal. Biopac MP100WSW Data Acquisition System was synchronized with the BioPlux research.

A respiratory pressure meter MicroRPM (CareFusion Corporation, San Diego CA, United States of America) was used to assess the maximal expiratory pressure (MEP). This quasi-static maximal manoeuvre was used to normalize the SEMG signal of abdominal muscles (maximal muscle activity of each muscle during breathing). A bacterial filter AFT1, mouthpiece AFT2 and nose clip AFT3 were also used.

\section{Procedures}

\section{Sample selection and characterization}

An electronic questionnaire was delivered to all participants to verify the selection criteria and to collect sociodemographic information. Anthropometric measures were assessed in participants who met the participation criteria. Height $(\mathrm{m})$ and body mass $(\mathrm{kg})$ - were measured using a seca 222 stadiometer, with a precision of $1 \mathrm{~mm}$ (technical data of enterprise), and a seca 760 scale, with a precision of $1 \mathrm{~kg}$ (technical data of enterprise), respectively. Then, body mass index was calculated. To assess postural asymmetries, the lower limb length $(\mathrm{cm})$ was measured using a seca 201 tape, with a precision of $1 \mathrm{~mm}$ (technical data of enterprise) (seca - Medical Scales and Measuring Systems, Hamburg, Germany), and the postural assessment was performed. These evaluations were performed to select the final sample. Women who were in luteal phase were contacted later for data collection.

\section{Data collection protocol}

The study procedures took place at a biomechanical laboratory and were performed in a controlled environment. To avoid inter-rater error, each researcher was responsible for only one task.

To perform the SEMG, the hair was shaved and an abrasive cream was used to remove the dead cells from the skin's surface. Skin was then cleaned with isopropyl alcohol (70\%), removing its oiliness and holding the dead cells. An electrode impedance checker was used to make sure that the impedance levels were below $5 \mathrm{~K} \Omega$, thus ensuring a good acquisition of sEMG signal (Hermens et al., 2000). The self-adhesive electrodes were placed with participants in standing position, $5 \mathrm{~min}$ after the skin preparation. These electrodes were placed parallel to the muscle fibre orientation, according to the references described in Table 2 (Criswell, 2011; Marshall and Murphy, 2003). The electrode placements were confirmed by palpation and muscle contraction. The reference electrode was placed in the anterior superior iliac spine of the contralateral hand dominant side. All electrodes were tested to control the cross-muscular signal (cross-talk), electrical noise and other interferences of sEMG signal (Hermens et al., 2000).

MEP was performed with the participant in standing, using a mouthpiece firmly held around the lips to prevent leakage and to support the cheeks, as well as a nasal clip to prevent the nasal breathing. To assess this manoeuvre, a forceful and maximal expiration was performed - the Valsalva manoeuvre - at total lung capacity. Each manoeuvre was encouraged verbally. These manoeuvres were performed during a 6-s period, with a resting time of 3 min. To normalize the sEMG signal of abdominal muscles, three reproducible manoeuvres were selected, according to

Table 2 Recommendations for the electrode placements of rectus abdominis (RA), external oblique (OE), transversus abdominis/internal oblique ( $\operatorname{Tr} \mathrm{A} / \mathrm{IO})$ and erector spinae (ES) muscles.

\begin{tabular}{ll}
\hline Muscle & Anatomical landmarks \\
\hline RA & $\begin{array}{l}2 \mathrm{~cm} \text { lateral to umbilicus, over the muscle mass } \\
\text { EO }\end{array}$ \\
TrAt/IO & $\begin{array}{l}\text { oblique angle } \\
2 \mathrm{~cm} \text { medially and below to the anterior superior iliac spine. } \\
\text { In this local, TrA and inferior } 10 \text { muscle fibres are mixed, so it is impossible to distinguish the surface } \\
\text { electromyographic activity of both. }\end{array}$ \\
ES & $2 \mathrm{~cm}$ lateral to spine, at L3 vertebra, over the muscle mass
\end{tabular}


American Thoracic Society/European Respiratory (2002) standards.

In standing, the maximal isometric voluntary contraction (MIVC) of ES muscle was performed to normalize data. The participant performed a trunk extension against an inelastic band placed on the scapular region. Three MIVC were performed, each one a 6 -s period, with a resting time of $3 \mathrm{~min}$.

Each participant breathing in supine, standing, 4-point kneeling and tripod positions, in a single data collection moment. The order of postural sets was randomized. In supine and standing, the participant had the upper limbs along the body, with feet shoulder-width apart and knees in loose pack position. In 4-point kneeling position, the participant was in triple flexion of lower limbs (hip and knee at $90^{\circ}$ ), with hands shoulder-width apart and elbows in loose pack position. In tripod position, the participant was sitting, with $45^{\circ}$ of trunk flexion to vertical, $90^{\circ}$ of hip flexion and upper limbs supported on a table. All joint amplitudes were confirmed using the Bubble ${ }^{\circledR}$ Inclinometer (trunk amplitude) and Baseline ${ }^{\circledR}$ Plastic Goniometer 360 Degree Head (hip and knee amplitudes), both with a precision of $1^{\circ}$ (technical data of enterprise). The respiratory flow transducer was kept perpendicular to the participant during all tasks. A single repetition of each task was performed for ten consecutive respiratory cycles, with a resting time of $3 \mathrm{~min}$. The respiratory rhythm (inspiratory time: $2 \mathrm{~s}$; expiratory time: $4 \mathrm{~s}$ ) was marked through a recorded voice. The participant experienced this respiratory rhythm prior to data collection.

After data collection, the electrodes were removed and a moisturizing cream was applied.

\section{Data processing}

A routine was developed in MatLab Student software (MathWorks, Pozuelo de Alarcon, Spain) to synchronize and process data. Firstly, the sEMG signal was converted into volts. A 2nd order digital filter Infinite Impulse Response Butterworth, one of $20 \mathrm{~Hz}$ (high pass) and another of $500 \mathrm{~Hz}$ (low pass), was applied to the SEMG signal to remove the electrical noise and/or cable movement; and, finally, a 2nd order digital filter Infinite Impulse Response - Butterworth of $30 \mathrm{~Hz}$ (high pass) was applied to remove the cardiac signal. Root mean square (RMS) to 10 samples was then calculated.

Acqknowledge software, version 4.1, was used to analyse data. The abdominal muscle activity was analysed during inspiration and expiration, independently. Both breathing phases were determined through the respiratory flow transducer signal. For the ten respiratory cycles collected, the mean RMS of four central respiratory cycles of each muscle was analysed during each task, with a posterior analysis of its average.

The muscle activity collected during the MEP manoeuvre was used to normalize the data related to the abdominal muscles. The mean RMS of three central seconds of the expiratory phase of each muscle was analysed, and then the average of the mean RMS of three reproducible manoeuvres was calculated. The percentage of activation intensity of each muscle was determined according to the following equation: $\begin{aligned} \text { Muscle activation intensity }(\%) & =\left(\frac{\text { mean } R M S \text { of each task }}{R M S \text { of the MEP }}\right) \\ & * 100\end{aligned}$

Breathing is a bilateral task. Accordingly, the global analysis of activation intensity of the RA, EO and TrA/IO muscles was considered during each breathing phase. For that, the average of the percentage of muscle activation intensity of the two hemi-trunks was calculated.

ES muscle activity was continuously analysed during breathing. For the ten respiratory cycles collected, the mean RMS of four central respiratory cycles was analysed during each task, with a posterior analysis of its average. The muscle activity collected during the MIVC manoeuvre was determined to normalize the data related to the ES muscle. The mean RMS of three central seconds was analysed, and then the average of the mean RMS of three repeated manoeuvres was calculated. The percentage of muscle activation intensity was determined according the following equation:

$$
\begin{aligned}
\text { Muscle activation intensity }(\%) & =\left(\frac{\text { mean } R M S \text { of each task }}{R M S \text { of the MIVC }}\right) \\
& * 100
\end{aligned}
$$

As described above, the global analysis of ES muscle activation intensity was considered during breathing. For that, the average of the percentage of muscle activation intensity of the two hemi-trunks was calculated.

\section{Statistical analysis}

IBM SPSS Statistics ${ }^{\circledR}$ software, version 20.0, (IBM Corporation, Armonk NY, United States of America) was used for the descriptive and inferential data analysis, with a significance level of 0.05 . Shapiro-Wilk test was used to test the normality of the data. Central tendency (mean) and dispersion (standard deviation) measures were used for the descriptive statistics. Repeated Measures Analysis of Variance was used to compare the percentage of muscle activation intensity between the different evaluation tasks (four postural sets), during inspiration and expiration. Bonferroni correction was used for the post-hoc analysis (Marôco, 2014).

\section{Results}

\section{ES muscle activation intensity}

ES muscle activation intensity was significantly greater in standing when compared to 4-point kneeling $(p=0,006)$, tripod $(p=0.019)$ and supine $(p=0.023)$ positions (see Fig. 1).

\section{Abdominal muscles activation intensity}

\section{Standing versus supine}

During expiration, RA muscle activation intensity was significantly greater in standing when compared to supine $(p=0.003)$ (see Fig. 3$)$. The activation intensity of EO $(p \leq 0.001)$ and TrA/IO $(p<0.001)$ muscles was significantly greater in standing when compared to supine, during both inspiration and expiration (see Figs. 2 and 3). 


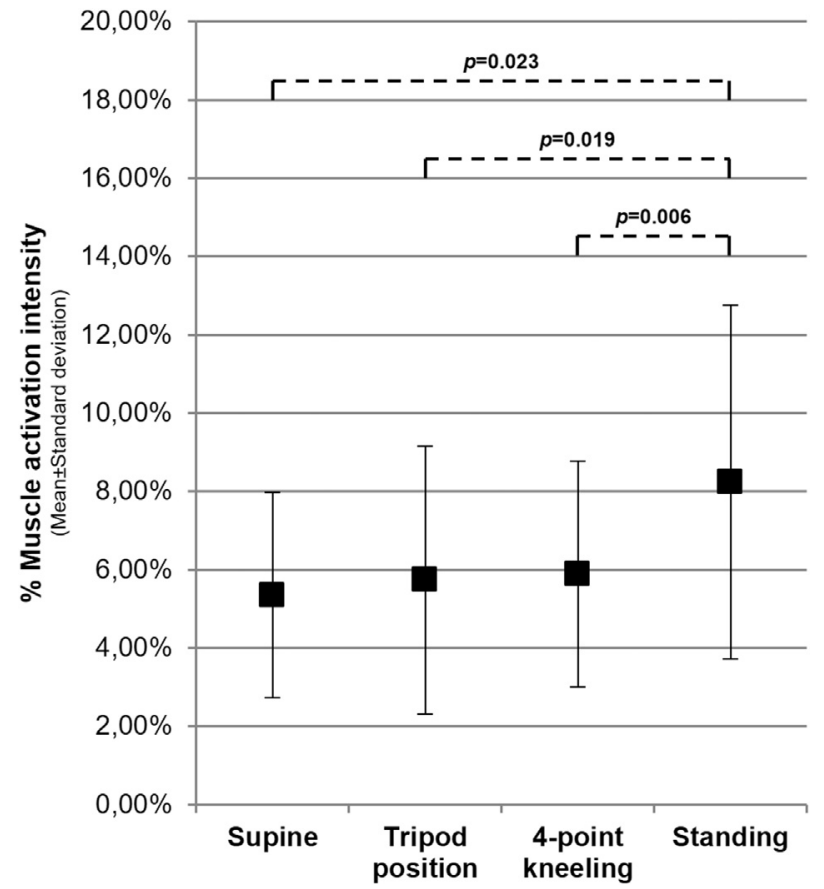

Figure 1 Activation intensity of erector spinae muscle (expressed as \%) during breathing in supine, tripod position, 4-point kneeling and standing. Data are presented as mean and standard deviation. $p$ values for significant differences between postural sets are also presented.

Tripod position versus standing and supine

During expiration, RA muscle activation intensity was significantly lower in tripod position when compared to standing ( $p=0.042)$ (see Fig. 3$)$. The activation intensity of EO (Inspiration: $p=0.003$; Expiration: $p<0.001$ ) and TrA/IO $(p<0.001)$ muscles was significantly lower in tripod position when compared to standing, during both inspiration and expiration (see Figs. 2 and 3 ).

During both breathing phases, TrA/IO muscle activation intensity was significantly greater in tripod position when compared to supine $(p \leq 0.001)$ (see Figs. 2 and 3 ).

\section{4-Point kneeling versus standing and supine}

During expiration, RA muscle activation intensity was significantly lower in 4-point kneeling position when compared to standing $(p=0.029)$ (see Fig. 3). TrA/IO muscle activation intensity was significantly lower in 4-point kneeling position when compared to standing $(p<0.001)$, during both inspiration and expiration (see Figs. 2 and 3 ).

During both breathing phases, the activation intensity of EO (Inspiration: $p=0.017$ and Expiration: $p=0.002$ ) and TrA/IO $(p<0.001)$ muscles was significantly greater in 4-point kneeling position when compared to supine (see Figs. 2 and 3 ).

\section{Tripod position versus 4-point kneeling}

During both inspiration and expiration, no significant differences were found in the activation intensity of any of the abdominal muscles between 4-point kneeling and tripod positions (see Figs. 2 and 3).

\section{Discussion}

The present study showed that the activation intensity of all abdominal muscles was higher in standing than in the other postural sets, during both inspiration and expiration. In tripod position and 4-point kneeling, TrA/IO muscle activation intensity was higher than in supine and lower than in standing, during both breathing phases. These data suggested that the abdominal muscles are important to the breathing mechanics, as well as postural control.

ES muscle activation intensity was measured. Different postural sets and functional goals (such as respiration) require that the CNS appropriately adjusts the postural muscle tone to gravity and changes in the base of support. The muscles which counteract the force of gravity, as ES muscle, should be more or less stiff or compliant to enable appropriate alignment for both stability and movement (Meadows and Williams, 2009; Mihailoff and Haines, 2013). The outcomes of this study indicated that the ES muscle activation intensity in standing was greater than in supine. In fact, the human skeletal motor system, due to the high

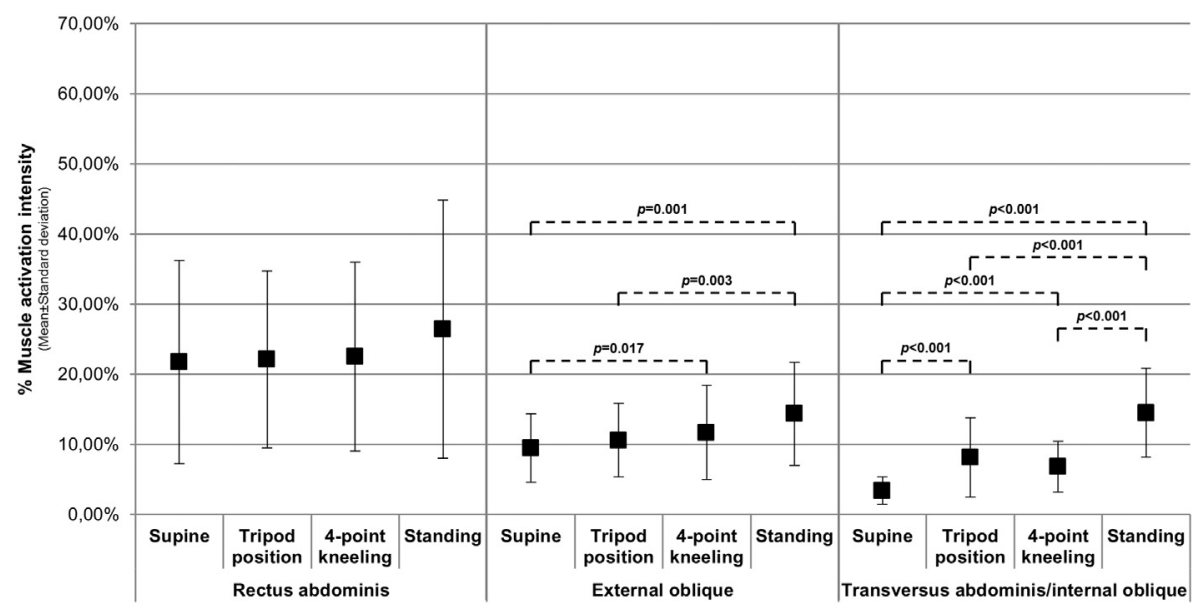

Figure 2 Activation intensity of rectus abdominis, external oblique and transversus abdominis/internal oblique muscles (expressed as \%) during inspiration in supine, tripod position, 4-point kneeling and standing. Data are presented as mean and standard deviation. $p$ values for significant differences between postural sets are also presented. 


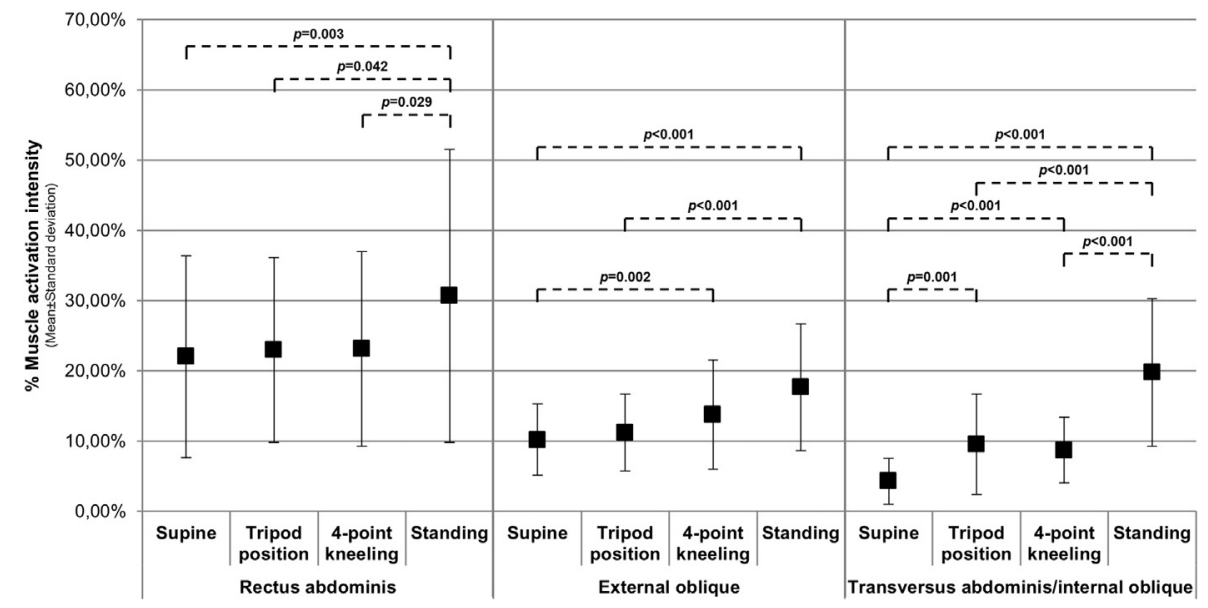

Figure 3 Activation intensity of rectus abdominis, external oblique and transversus abdominis/internal oblique muscles (expressed as \%) during expiration in supine, tripod position, 4-point kneeling and standing. Data are presented as mean and standard deviation. $p$ values for significant differences between postural sets are also presented.

position of the centre of mass regarding the small size of base of support, is poorly adapted to the preservation of a vertical position (standing) (Hodges et al., 2002). Unlike supine, the gravitational pull would be increased in standing, resulting in greater feedback from the stretch receptors of ES muscle, thus raising motor-neuron pool excitability and increasing muscle recruitment (Meadows and Williams, 2009; Mihailoff and Haines, 2013).

Postural sets, which require the maintenance of a static joint orientation of the spine, generally involve the coactivation of antigravity muscles and their antagonist muscles (abdominal muscles). In standing, the combination of a greater extensor moment (from ES muscle) and an opposing greater flexor moment (from abdominal muscles) tonically increases intra-abdominal pressure, and, consequently, unloads compressive forces on the spine (Cholewicki et al., 1999). Therefore, in the present study, a greater activation intensity of all abdominal muscles was observed in standing when compared to supine, which supports their primary postural function, increasing the postural tone when the challenge to stability is increased. Nevertheless, the worse breathing mechanics in supine may be explained by the lower recruitment of abdominal muscles. Although the elastic recoil for the lung is relatively unchanged due to the action of gravity, the chest wall mechanics, namely diaphragm muscle, is affected. In standing, the abdominal content is being pulled away from the diaphragm muscle. As opposed, in supine it is pushing inward against the relaxed diaphragm muscle, decreasing the overall outward recoil of the chest wall, and so the functional residual capacity (Levitzky, 2013). Thus, from standing to supine, the increased abdominal muscle compliance allows the resistance provided by the abdominal content to the diaphragm muscle descent is less effective in expanding the lower rib cage (Strohl et al., 1984). The results of the present study were consistent with earlier studies of Abe et al. (1996), Barrett et al. (1994) and De Troyer (1983). Mew (2009) also found a greater resting recruitment (thickness) of all abdominal muscles in standing when compared to supine, using ultrasound imaging. Nevertheless, Kera and Maruyama (2005) reported only a greater IO muscle activity in standing when compared to supine during spontaneous breathing. However, these authors did not normalize data to account for submaximal/maximal voluntary contraction.

The outcomes of this study indicated that the activation intensity of abdominal muscles in tripod position was lower than in standing and greater than in supine. The tripod position is often adopted as a result of breathlessness. Patients in respiratory distress lean forward with hands supporting them on their knees or forearms to stabilize and elevate the shoulder girdle (Bott et al., 2009). Consequently, this postural set improves the length-tension relationship of muscles that are attached between the ribs and upper limb or shoulder girdle, and so their capacity to act as accessory muscles of the breathing (Banzett et al., 1988). Moreover, this lean-forward position in sitting, with the passive fixing of shoulder girdle, reduces the postural load, resulting in lower ES muscle activation intensity in tripod position when compared to standing. In fact, as explained above, the gravitational pull would be reduced in tripod position, decreasing abdominal muscle recruitment. Furthermore, unlike supine, the downward and outward displacement of abdominal content in tripod position (Dean, 1985) may place the abdominal muscles in an improved position for contraction with some degree of lean forward. TrA muscle, due to its circumferential arrangement, has the most appropriate mechanical efficiency, which makes it easier to recruit into this postural set (De Troyer et al., 1990). Consequently, the tripod position may help to dome the diaphragm muscle, lengthening the muscle fibres, improving the length-tension relationship, and therefore its force and respiratory capacity (Barach, 1974). Thus, the improvement in breathing mechanics in this postural set may be explained by the higher TrA/IO muscle recruitment. There is little evidence regarding individual recruitment of abdominal muscles during breathing in forward lean positions. Nevertheless, Kera and Maruyama (2005) reported significantly higher muscle activity only for the EO muscle in sitting-with-elbow-onthe-knee when compared to supine. This recruitment may be explained by differences in setting between the 
sitting-with-elbow-on-the-knee and the tripod position, wherein in the last position there is a passive fixing of the shoulder girdle.

As in tripod position, a large base of support, which reduces the postural load, characterizes the 4-point kneeling position. Therefore, in this study, lower ES muscle activation intensity was observed in 4-point kneeling position when compared to standing, resulting in lower recruitment of abdominal muscles, as previously discussed. Moreover, the 4-point kneeling position, as well as the tripod position, allows the abdominal muscles to sag, facilitating a stretch (Norris, 1999). This postural set is likely to increase the feedback from the muscle stretch receptors, thus raising the motor-neuron pool excitability of the TrA/IO muscle (Beith et al., 2001). However, the gravitational stretch may be increased in 4-point kneeling position when compared to tripod position, which implies a more demanding TrA/IO muscle recruitment in isolation. This requirement is likely to increase the need for EO muscle recruitment to assist in postural and respiratory functions. Therefore, in the present study, the activation intensity of EO and $\operatorname{TrA} / 10$ muscles in 4-point-kneeling position was greater than in supine. EO and TrA/IO muscles share the same fibro-osseous attachments to the costal cartilages, the thoracolumbar fascia, the iliac crest and the pubis (Drake et al., 2014), whereby these muscles together flatten the abdomen in 4-point kneeling position.

The postural load and the gravitational stretch in both tripod and 4-point-kneeling positions allow forward movement of the abdominal content, out of the way of the diaphragm muscle. In this study, there were no significant differences in the activation intensity of all abdominal muscles between tripod position and 4-point kneeling. Thus, it is theorized that, in these postural sets, abdominal muscle recruitment, namely the TrA/IO muscle, may be important to thoracic-abdominal movement improvement. Accordingly, further investigation is needed in order to understand the impact of these postural sets or other abdominal muscle recruitment strategies, such as hollowing or bracing, on breathing mechanics and pattern.

None of the participants in the present study suffered from chronic respiratory pathologies. Chronic obstructive pulmonary disease may alter the patient's ability to recruit their abdominal muscles to the breathing mechanics. Therefore, the results of this study suggested that the tripod position often assumed by the breathless patients, as well as 4-point kneeling position, can have an impact on the enablement of abdominal muscle activation. However, further studies conducted among chronic obstructive pulmonary disease patients are needed.

\section{Conclusion}

In healthy subjects, the change of body orientation promoted different impact on abdominal muscle activity during breathing. The highest activation intensity of abdominal muscles was observed in standing as opposed to supine. Postural load and gravitational stretch are factors that should be considered in relation to the specific recruitment of abdominal muscles for breathing mechanics. In tripod position and 4-point kneeling, TrA/IO muscle activation intensity may be a determining factor to improve the breathing mechanics. Thus, TrA/IO muscle recruitment seems to be important for the synchronization of postural and respiratory functions.

\section{References}

Abe, T., Kusuhara, N., Yoshimura, N., Tomita, T., Easton, P.A., 1996. Differential respiratory activity of four abdominal muscles in humans. J. Appl. Physiol. (1985) 80, 1379-1389.

American Thoracic Society/European Respiratory S, 2002. ATS/ERS Statement on respiratory muscle testing. Am. J. Respir. Crit. Care Med. 166, 518-624.

Balasubramaniam, R., Wing, A.M., 2002. The dynamics of standing balance. Trends Cogn. Sci. 6, 531-536.

Banzett, R.B., Topulos, G.P., Leith, D.E., Nations, C.S., 1988. Bracing arms increases the capacity for sustained hyperpnea. Am. Rev. Respir. Dis. 138, 106-109.

Barach, A.L., 1974. Chronic obstructive lung disease: postural relief of dyspnea. Arch. Phys. Med. Rehabil. 55, 494-504.

Barrett, J., Cerny, F., Hirsch, J.A., Bishop, B., 1994. Control of breathing patterns and abdominal muscles during graded loads and tilt. J. Appl. Physiol. (1985) 76, 2473-2480.

Beith, I.D., Synnott, R.E., Newman, S.A., 2001. Abdominal muscle activity during the abdominal hollowing manoeuvre in the four point kneeling and prone positions. Man. Ther. 6, 82-87.

Booth, S., Burkin, J., Moffat, C., Spathis, A., 2014. Positions to Ease Breathlessness, Managing Breathlessness in Clinical Practice. Springer, London, pp. 49-65.

Bott, J., Blumenthal, S., Buxton, M., Ellum, S., Falconer, C., Garrod, R., Harvey, A., Hughes, T., Lincoln, M., Mikelsons, C., Potter, C., Pryor, J., Rimington, L., Sinfield, F., Thompson, C., Vaughn, P., White, J., British Thoracic Society Physiotherapy Guideline Development G, 2009. Guidelines for the physiotherapy management of the adult, medical, spontaneously breathing patient. Thorax 64 (Suppl. 1), i1-51.

Chanthapetch, P., Kanlayanaphotporn, R., Gaogasigam, C., Chiradejnant, A., 2009. Abdominal muscle activity during abdominal hollowing in four starting positions. Man. Ther. 14, 642-646.

Cholewicki, J., Juluru, K., McGill, S.M., 1999. Intra-abdominal pressure mechanism for stabilizing the lumbar spine. J. Biomech. 32, 13-17.

Criswell, E., 2011. Cram's Introduction to Surface Electromyography, second ed. Jones \& Bartlett Publishers, Sudbury, MA.

De Troyer, A., 1983. Mechanical role of the abdominal muscles in relation to posture. Respir. Physiol. 53, 341-353.

De Troyer, A., Estenne, M., 1988. Functional anatomy of the respiratory muscles. Clin. Chest Med. 9, 175-193.

De Troyer, A., Estenne, M., Ninane, V., Van Gansbeke, D., Gorini, M., 1990. Transversus abdominis muscle function in humans. J. Appl. Physiol. (1985) 68, 1010-1016.

Dean, E., 1985. Effect of body position on pulmonary function. Phys. Ther. 65, 613-618. 
Drake, R., Vogl, A.W., Mitchell, A.W.M., 2014. Abdomen, Gray's Anatomy for Students, third ed. Churchill Livingstone/Elsevier, Philadelphia, PA, pp. 253-420.

Estenne, M., Yernault, J.C., De Troyer, A., 1985. Rib cage and diaphragm-abdomen compliance in humans: effects of age and posture. J. Appl. Physiol. (1985) 59, 1842-1848.

Goldman, J.M., Lehr, R.P., Millar, A.B., Silver, J.R., 1987. An electromyographic study of the abdominal muscles during postural and respiratory manoeuvres. J. Neurol. Neurosurg. Psychiatry 50, 866-869.

Hermens, H.J., Freriks, B., Disselhorst-Klug, C., Rau, G., 2000. Development of recommendations for SEMG sensors and sensor placement procedures. J. Electromyogr. Kinesiol 10, 361-374.

Hides, J., Richardson, C., Hodges, P., 2004. Local Segmental Control, Therapeutic Exercise for Lumbopelvic Stabilization, second ed. Churchill Livingstone, Edinburgh, pp. 185-219.

Hodges, P.W., 1999. Is there a role for transversus abdominis in lumbo-pelvic stability? Man. Ther. 4, 74-86.

Hodges, P.W., Butler, J.E., McKenzie, D.K., Gandevia, S.C., 1997. Contraction of the human diaphragm during rapid postural adjustments. J. Physiol. 505 (Pt 2), 539-548.

Hodges, P.W., Gandevia, S.C., 2000a. Activation of the human diaphragm during a repetitive postural task. J. Physiol. 522 (Pt 1), 165-175.

Hodges, P.W., Gandevia, S.C., 2000b. Changes in intra-abdominal pressure during postural and respiratory activation of the human diaphragm. J. Appl. Physiol. (1985) 89, 967-976.

Hodges, P.W., Gurfinkel, V.S., Brumagne, S., Smith, T.C., Cordo, P.C., 2002. Coexistence of stability and mobility in postural control: evidence from postural compensation for respiration. Exp. Brain Res. 144, 293-302.

Hodges, P.W., Heijnen, I., Gandevia, S.C., 2001. Postural activity of the diaphragm is reduced in humans when respiratory demand increases. J. Physiol. 537, 999-1008.

Horak, F.B., Henry, S.M., Shumway-Cook, A., 1997. Postural perturbations: new insights for treatment of balance disorders. Phys. Ther. 77, 517-533.

Kera, T., Maruyama, H., 2005. The effect of posture on respiratory activity of the abdominal muscles. J. Physiol. Anthropol. Appl. Hum. Sci. 24, 259-265.

Lackner, J.R., DiZio, P., 2005. Vestibular, proprioceptive, and haptic contributions to spatial orientation. Annu. Rev. Psychol. 56, 115-147.

Levitzky, M.G., 2013. Mechanics of Breathing, Pulmonary Physiology. McGraw-Hill Education/Medical, New York, NY, pp. 12-57.

Marôco, J., 2014. Análise Estatística com o SPSS Statistics, sixth ed. ReportNumber, Lda., Pero Pinheiro.

Marshall, P., Murphy, B., 2003. The validity and reliability of surface EMG to assess the neuromuscular response of the abdominal muscles to rapid limb movement. J. Electromyogr. Kinesiol 13, 477-489.

Meadows, L., Williams, J., 2009. An understanding of Functional movement as a basis for clinical reasoning. In: Raine, S., Meadows, L., Lynch-Ellerington, M. (Eds.), Bobath Concept: Theory and Clinical Practice in Neurological Rehabilitation. Wiley-Blackwell, Oxford, pp. 23-42.
Mew, R., 2009. Comparison of changes in abdominal muscle thickness between standing and crook lying during active abdominal hollowing using ultrasound imaging. Man. Ther. 14, 690-695.

Mihailoff, G.A., Haines, D.E., 2013. Motor system I: peripheral sensory, brainstem, and spinal influence on anterior horn neurons. In: Haines, D.E. (Ed.), Fundamental Neuroscience for Basic and Clinical Applications. Elsevier/Saunders, Philadelphia, PA, pp. 324-337.

Norris, C., 1999. Functional load abdominal training: part 2. J. Bodyw. Mov. Ther. 3, 208-214.

Reeve, A., Dilley, A., 2009. Effects of posture on the thickness of transversus abdominis in pain-free subjects. Man. Ther. 14, 679-684.

Strohl, K.P., Mead, J., Banzett, R.B., Lehr, J., Loring, S.H., O'Cain, C.F., 1984. Effect of posture on upper and lower rib cage motion and tidal volume during diaphragm pacing. Am. Rev. Respir. Dis. 130, 320-321.

Thompson, P.D., 2014. Health appraisal and risk assessment. In: Pescatello, L.S., Arena, R., Riebe, D., Thompson, P.D. (Eds.), ACSM's Guidelines for Exercise Testing and Prescription, ninth ed. Wolters Kluwer/Lippincott Williams \& Wilkins Health, Philadelphia, pp. 1-18. 